\title{
A case of intravascular large B cell lymphoma presenting as nodular goiter

\begin{abstract}
Background: Intravascular large B-cell lymphoma (IVLBCL) is a subtype of diffuse large B-cell lymphoma (DLBCL) that is rare and highly aggressive and that may progressively involve many organs. CNS (central nervous system), BM (bone marrow) and skin are the most common systems involved. To date, only 2 cases of IVLBCL involving the thyroid have been reported.

Case presentation: Here, we report a case of IVLBCL involving the thyroid and accompanied by bilateral nodular goiter. In this case, a thyroid mass was identified in a physical examination of a 68-year-old male who initially presented with dyspnea accompanied by intermittent headache for approximately 1 month. Computed tomography scans revealed that the left lobar thyroid was occupied by a large, slightly lower density mass $(5.8 \times 4.7 \times 8.4 \mathrm{~cm})$. However, the patient had no hyperthyroidism or hoarseness. Levels of thyroid hormones and anti-thyroid autoantibodies in the serum were normal preoperatively. Thyroid mass resection was performed to establish a diagnosis and to relieve symptoms.

Conclusions: Pathological results of the surgical specimen revealed that large atypical lymphoma cells filled the capillaries in the lesion area. Immunohistochemical staining revealed that the large-sized tumor cells were positive for CD20, PAX-5, MUM-1 and BCL-2, and were negative for CD3, CD5, CD43, CD10, CD23, CyclinD1, CD138, CD30, ALK, CD56, MPO, S-100, TTF-1, TG (thyroglobulin) and CT (calcitonin). The Ki-67 index was estimated to be approximately $85 \%$. The patient was subsequently diagnosed as "Classical" IVLBCL non-germinal center B-cell type. The patient declined chemotherapy and died in the fifth month after operation.
\end{abstract}

Keywords: Intravascular lymphoma, Large B-cell, Thyroid, Nodular goiter

\section{Background}

Intravascular large B-cell lymphoma (IVLBCL) is a rare and highly aggressive subtype of extranodal DLBCL with an estimated annual incidence of fewer than 0.5 cases per $1,000,000[1,2]$. IVLBCL was first described by Pfleger and Tappeiner [3] in 1959 and is characterized by the proliferation of malignant B-cells in small- and medium-size vessels. According to patients' initial clinical presentation and bone marrow biopsy, the clinical phenotypes of IVLBCL are mainly classified into "Classical" IVLBCL and "Asian variant" IVLBCL [4-6]. "Classical” IVLBCL is characterized by CNS and/or cutaneous involvement. "Asian

\footnotetext{
* Correspondence: luobo198705051@126.com

${ }^{\dagger}$ Equal contributors

'Department of Pathology, The Central Hospital of Wuhan, Tongji Medical College, Huazhong University of Science and Technology, No. 26, Shengli Street, Wuhan City, Hubei Province 430014, People's Republic of China Full list of author information is available at the end of the article
}

variant" IVLBCL is characterized by hemophagocytic syndrome, $\mathrm{BM}$ involvement, fever and hepatosplenomegaly.

IVLBCL can progressively involve any organ of the body [7-9]. In 2014, Fonkem et al. [2] retrospectively analyzed 740 cases of intravascular lymphoma (IVL) reported in the literature published worldwide between 1959 and 2011, among which 651 were IVLBCL. This retrospective research found that $\mathrm{CNS}, \mathrm{BM}$, spleen, skin, and lung were the most common systems involved, accounting for $60 \%, 11,8$, and $7 \%$ of cases, respectively. The involvement of other organs, such as the kidney, ovaries, uterus, and adrenal glands, has also been reported [10-12]. To our knowledge, only 2 cases of IVLBCL that involve the thyroid have been reported in the literature to date $[13,14]$. One of these cases was a 68-year-old male who was admitted to the neurology department due to vertigo. A thyroid ultrasound revealed a left-sided nodule, and fine-needle aspiration cytology results revealed a papillary carcinoma in the left-sided 
nodule. Subsequently, the patient underwent a total thyroidectomy, and the histopathologic results revealed that IVLBCL presented as a dominant component [13]. The clinical-pathological details of the other case are unknown [14]. Here, we report a case of IVLBCL involving the thyroid. In this case, a 68-year-old male initially presented with dyspnea accompanied by intermittent headaches.

\section{Case presentation \\ Clinical history}

The 68-year-old male patient had a history of hypertension for approximately 20 years and chronic bronchitis for approximately 10 years. The patient was admitted to the respiratory department of our hospital because he had suffered from dyspnea and intermittent headaches for approximately 1 month. Physical examination revealed a $7 \times 6 \mathrm{~cm}$ non-tender mass in the neck. No signs of hyperthyroidism or hoarseness existed. Neurological examination revealed no positive signs. Blood pressure $(110 / 70 \mathrm{mmHg})$ was within the normal range. There was no family history of thyroid disease.

On admission, laboratory examination revealed the following: erythrocytes $3.94\left(4.3-5.8 \times 10^{12} / \mathrm{L}\right)$, hemoglobin content $124(130-175 \mathrm{~g} / \mathrm{L})$, serum lactate dehydrogenase (LDH) 480.0 (15-240 U/L), hydroxybutyrate dehydrogenase $311.0(50-220 \mathrm{U} / \mathrm{L})$, total bilirubin $30.1 \quad(5.1-20 \mu \mathrm{mol} / \mathrm{L})$, direct bilirubin $11.6 \quad(0.1-$ $10 \mu \mathrm{mol} / \mathrm{L})$ and indirect bilirubin $18.1(3-15 \mu \mathrm{mol} / \mathrm{L})$. All numbers in parentheses mentioned above indicate the reference range. Thyroid function tests revealed that serum calcitonin, thyroid hormones, thyroid stimulating hormone, and anti-thyroid autoantibodies were normal.

A CT (computed tomography) scan of the chest demonstrated bronchitis and bilateral bullae of lung. A CT scan of the head and neck revealed the following: 1) A slightly higher density nodule $(1.5 \mathrm{~cm})$ in the cerebellum caused a slight space-occupying effect; 2) Thyroid neoplasm invaded the anterior superior mediastinum. The left lobar thyroid was enlarged and occupied by a large, slightly lower density mass $5.8 \times 4.7 \times 8.4 \mathrm{~cm}$ in size. The mass protruded down to the chest entrance level, and the main manifestations were scattered calcified lesions, flaky necrosis areas of low density, and inhomogeneous enhancement (Fig. 1a). In the right lobar thyroid, there was a slightly higher density of round nodules $(1.3 \times 1.2 \mathrm{~cm})$ without calcification or significant enhancement. The trachea was pushed by the giant mass to the right side and became narrow (Fig. 1b). There were no abnormal visible lymph nodes in either side of the neck. Thyroid color Doppler ultrasound revealed a giant hypoechoic mass $(7.1 \times 4.7 \times 2.4 \mathrm{~cm})$ with clear separation and heterogeneous echotexture in the left lobar thyroid and a hybrid echo-mass $(1.3 \times 1.2 \times 1.0 \mathrm{~cm})$ in the right side.

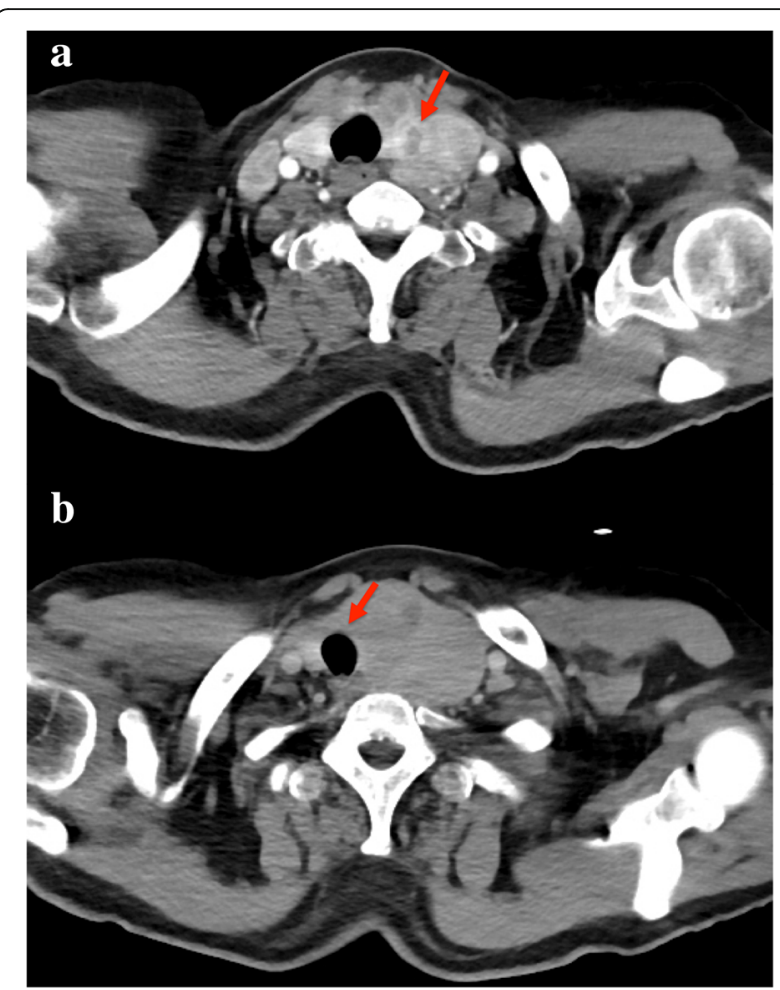

Fig. 1 Computed tomography scan of the head and neck. a The left lobar thyroid is enlarged and is occupied by a large slightly lower density mass $(5.8 \times 4.7 \times 8.4 \mathrm{~cm})$. The mass expanded down to the chest entrance level. The main manifestations are scattered calcified lesions, flaky necrosis areas of low density, and inhomogeneous enhancement. $b$ The trachea is pushed to the right by the remarkably enlarged thyroid

CDFI exhibited an obvious blood flow signal in the lower density mass.

The patient was transferred to the thyroid breast surgery department. Thyroid mass resection was performed to establish a diagnosis and to relieve the symptoms caused by tumor compression. During the operation, the markedly enlarged thyroid was easily observed, and the trachea was pushed to the right. Multiple masses of various sizes were noted in the bilateral thyroid. The largest of these masses $(10.0 \times 6.0 \times 5.0 \mathrm{~cm})$ was noted in the left lobar thyroid, and $2 / 5$ of the mass was behind the sternum with an intact capsule. In addition, a small $(1.8 \times 1.2 \times 1.0 \mathrm{~cm})$ mass with a distinct border was located in the middle of the right lobar thyroid. These masses were completely removed and sent to the pathology department for frozen section examination. Frozen section histological results revealed nodular goiter of bilateral and focal atypical hyperplasia in the left lobe. The patient then underwent a total thyroidectomy with central neck area lymph node dissection.

\section{Gross features}

The entire thyroid had a complete packing membrane and a dark red appearance. The cutting appearance of 
the thyroid revealed that the giant mass $(8.1 \times 5.0 \times 4.5 \mathrm{~cm})$ in the left lobe was solid with medium hard features and an uneven texture. In addition, the $1.8 \times 1.2 \times 1.0 \mathrm{~cm}$ mass in the right lobe was gelatinous, soft, and uniform in texture. Both masses had a complete capsule. No visible enlargement of the lymph nodes was detected in the central neck area. Twenty paraffin blocks were cut from the total thyroidectomy specimen, 15 of which were cut from the left lobe and 5 from the right. However, only 3 paraffin blocks in the left lobe contained tumor cells as demonstrated by subsequent microscopic examination.

\section{Histological features}

All tumor cells were located in the interstitium of thyroid follicular cells without forming a clear boundary line, which appeared as an invasion of lymphocytes under low magnification (Fig. 2a). Thus, it might have been easy to miss the tumor under low magnification if pathologists had not been informed of the gross features. However, these tumor cells could be distinguished from mature small lymphocytes under lower magnification mainly based on their intravascular growth pattern, atypical nuclei and larger size. Capillaries in the lesion area filled by large atypical lymphoma cells exhibited an expanded appearance (Fig. 2b-c), which was quite different from normal capillary appearance. Under higher magnification, large-sized tumor cells exhibit minimal cytoplasm, a thick nuclear membrane, irregular nuclear contours, and prominent nucleoli and coarse to somewhat dispersed chromatin (Fig. 2c-d).

\section{IHC}

Immunohistochemical staining revealed that the largesized tumor cells were positive for CD20, PAX-5, MUM1 and BCL-2, and were negative for CD3, CD5, CD43, CD10, CD23, CyclinD1, CD138, CD30, ALK, CD56, MPO, S-100, TTF-1, thyroglobulin and calcitonin. The Ki-67 index was estimated to be approximately $85 \%$. The intravascular growth pattern of the tumor cells was highlighted by CD34 staining of the endothelial cells (Fig. 3). CD20 and CD34 staining revealed that the tumor cells were not involved in the right lobe of the thyroid or the central neck area lymph nodes.

\section{Treatments and outcome}

Based on the clinical history and histopathological and immunohistochemistry results, the patient was diagnosed as "Classical" IVLBCL non-germinal center B-cell type. The patient was recommended to receive the BCL-6/BCL-2 and MYC gene re-arrangement test and 6 cycles of Rituximab plus cyclophosphamide, doxorubicin, vincristine and prednisolone (R-CHOP) immunochemotherapy. However, the patient declined chemotherapy and died in the fifth month after operation.

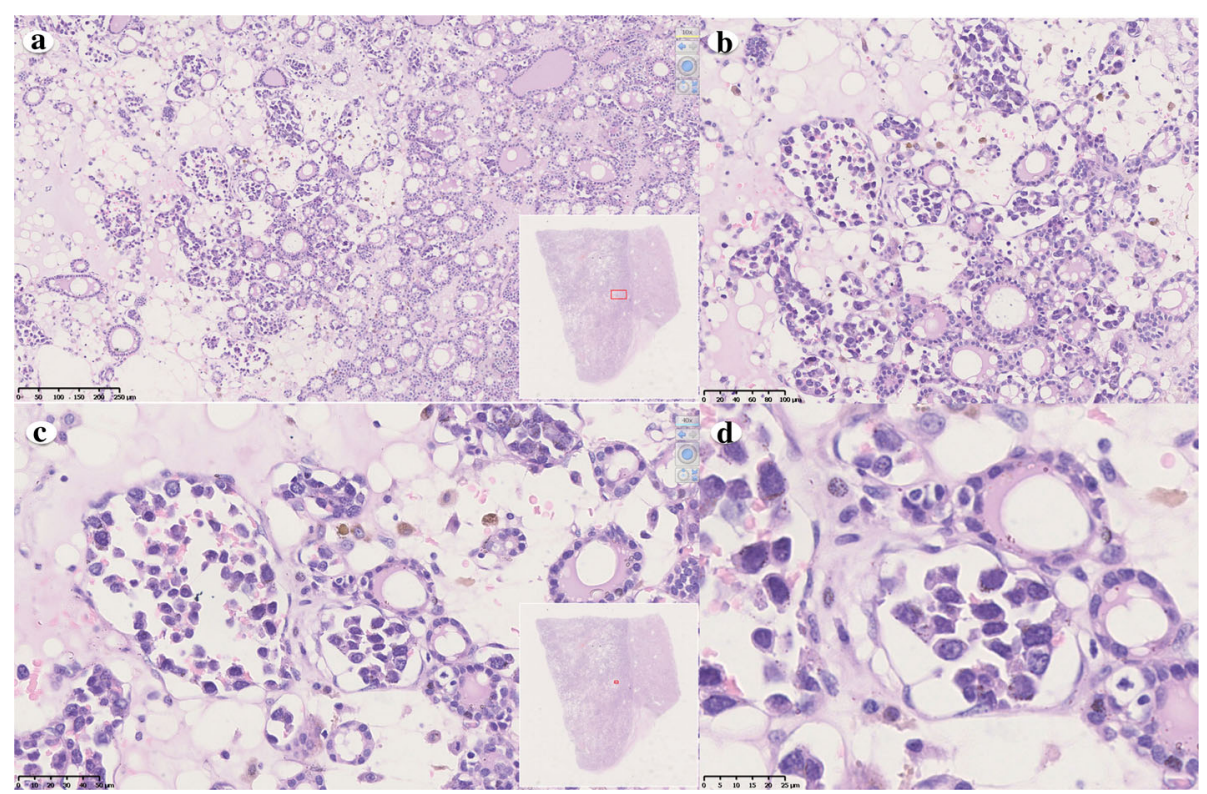

Fig. 2 Histopathological features of thyroid intravascular large B-cell lymphoma. a Tumor cells are located in the interstitial of thyroid follicular without forming a clear boundary line (original magnification $\times 100$ ). b, c Capillaries in the lesion area are filled by large atypical cells exhibiting an expansion appearance (original magnification $\times 200$ and $\times 400$ respectively). $d$ Large-sized tumor cells with minimal cytoplasm, a thick nuclear membrane, irregular nuclear contours, prominent nucleoli and coarse to somewhat dispersed chromatin are noted (original magnification $\times 400$ ) 


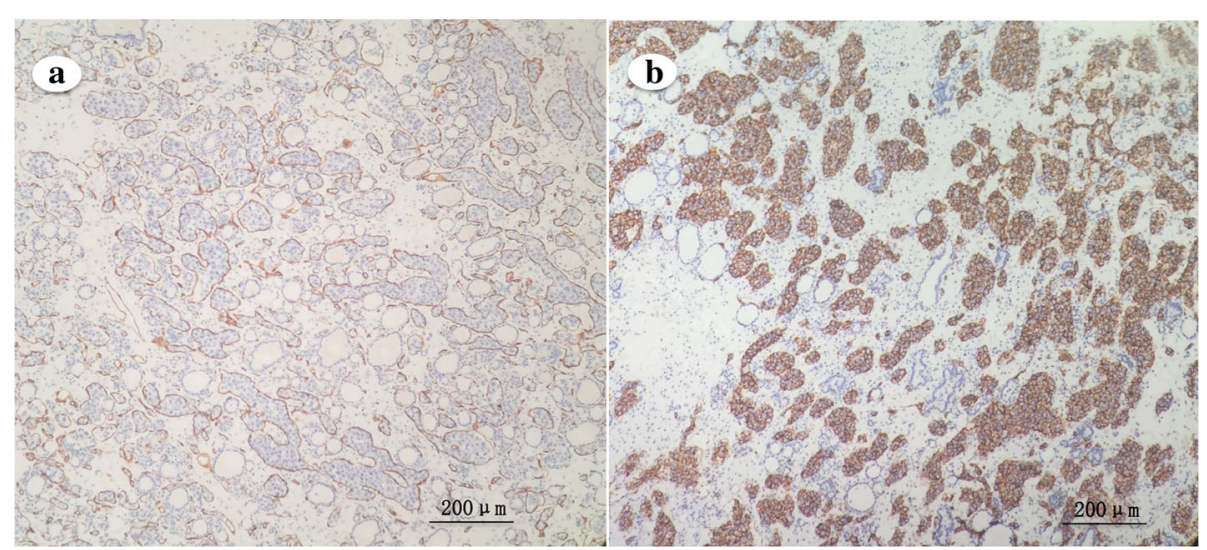

Fig. 3 Immunohistochemistry staining of thyroid intravascular large B-cell lymphoma. a CD20-positive staining confirms that the large atypical lymphoma originates from B-cells (original magnification $\times 100$ ). b CD34 immunohistochemistry staining highlights the intravascular growth pattern of tumor cells (original magnification $\times 100$ )

\section{Discussion}

IVLBCL is a rare malignant tumor that progresses rapidly and that may involve any organ, and IVLBCL involving the thyroid is particularly rare [2]. Depending on the organ involved, the clinical presentations of IVLBCL are non-specific and unpredictable, and may include neurological signs and symptoms, cutaneous lesions, fever, hepatosplenomegaly and cytopenia [5, 7, 15]. Given the high variance of clinical presentation, most patients undergo many investigations before the diagnosis is established. Occasionally, patients cannot be diagnosed or treated promptly as a result of the lack of sensitive diagnostic methods [5].

The patient in this case suffered from dyspnea and intermittent headaches for approximately 1 month. A giant non-tender mass in the neck was accidentally discovered upon physical examination. A CT scan demonstrated that the remarkably enlarged left lobar thyroid was occupied by a giant mass that suppressed the trachea. Thyroid function tests were normal preoperatively, whereas frozen section histological results revealed a nodular goiter with bilateral and focal atypical hyperplasia in the left lobe. Taking the increase in serum LDH and the occupied lesion in the cerebellum into consideration, we examined the tissue sections carefully. Eventually, we found lesion areas in three of the total 20 paraffin blocks. Thus, it is important to be aware of this entity and to examine the small vessels in biopsies as carefully as possible. In addition, the 18F-FDG PET/CT scan was demonstrated to have diagnostic value in IVLBCL $[16,17]$.

Histopathological differential diagnosis of IVLBCL mainly includes intravascular anastomotic large T-cell lymphoma [18], intravascular NK/T cell lymphoma [19], the other intravascular lymphoma with B-cell immunophenotype, such as exudative lymphoma, benign atypical intravascular CD30-positive T cell hyperplasia, and intravascular lymphatic tissue cell proliferation. Histopathological and immunohistochemistry staining results of this case were mostly typical, so no differential diagnosis was required. Regarding the clinical phenotype of the patient in our case, we might prefer the diagnosis of "Classical" IVLBCL to "Asian variant" IVLBCL, given that the patient did not experience fever, hepatosplenomegaly, hypoproteinemia or thrombopenia. The patient also had cerebellum involvement at the time of diagnosis according to medical history and $\mathrm{CT}$ results. Moreover, bone marrow biopsy is useful in making the correct classification of the clinical phenotype [20].

Due to the low morbidity of IVLBCL, prognostic factors of IVLBCL remain uncertain [21]. One study has suggested that a younger age (less than 70 years old), non-CNS site of initial diagnosis, $\mathrm{LDH}<700 \mathrm{U} / \mathrm{L}$ and rituximab treatment were favorable prognostic factors [2]. By contrast, patients with hemophagocytic syndrome or CNS disease have a poorer prognosis $[2,22]$. Other studies have reported that patients who had de novo CD5+ might present with aggressive disease, which signifies a very poor prognosis $[23,24]$. In addition, some other potential prognostic factors might be involved, such as general physical state and extra-vascular involvement. Although IVLBCL is known for its rapid progression, some studies have suggested that long-term remission in patients with IVLBCL would be observed if they were diagnosed early and treated with conventional R-CHOP in time $[15,25,26]$.

\section{Conclusions}

In summary, we have presented the clinical features and natural course of primary thyroid IVLBCL. The patient in this case was diagnosed late with thyroid and cerebellum involvement. This case report suggests that clinicians and 
pathologists should be aware of the existence of thyroid IVLBCL. In addition, early diagnosis of IVLBCL through biopsy and treatment of patients with $\mathrm{R}-\mathrm{CHOP}$ may be helpful to improve patients' survival.

\begin{abstract}
Abbreviations
ALK: Anaplastic lymphoma kinase; BM: Bone marrow; CD: Cluster of differentiation; CDFI: Color Doppler flow imaging; CNS: Central nervous system; CT: Computed tomography; DLBCL: Diffuse large B-cell lymphoma; IVL: Intravascular lymphoma; IVLBCL: Intravascular large B-cell lymphoma; LDH: Serum lactate dehydrogenase; MPO: Myeloperoxidase; MUM-1: Multiple myeloma oncogene 1; PAX-5: Paired box protein 5; R-CHOP: Rituximab plus cyclophosphamide, doxorubicin, vincristine, and prednisolone; S-100: S-100 protein; TTF-1: Thyroid transcription factor 1
\end{abstract}

\section{Acknowledgments}

We thank the Department of Pathology, the Union Hospital, Huazhong University of Science and Technology, Wuhan, China for assistance with pathological diagnosis.

\section{Funding}

Not applicable.

\section{Availability of data and materials}

Data sharing is not applicable to this article as no datasets were generated or analyzed during the current study.

\section{Author's contributions}

BL collected the clinical information on the patient, reviewed the pathologic specimen, and selected images. JL, WHL, YXS, PZ, YHX, and HFZ offered assistance in specimen evaluation and image selection. BL and JMC wrote the manuscript. All authors read and approved the final manuscript.

\section{Ethics approval and consent to participate}

The need for ethical approval was waived by the Institutional Ethics Committee of the Central Hospital of Wuhan, China.

\section{Consent for publication}

Written informed consent for the publication of clinical details and clinical images of the patient was obtained from a relative of the patient. A copy of the consent form is available for review by the editor of this journal.

\section{Competing interests}

The authors declare that they have no competing interests.

\section{Publisher's Note}

Springer Nature remains neutral with regard to jurisdictional claims in published maps and institutional affiliations.

\section{Author details}

'Department of Pathology, The Central Hospital of Wuhan, Tongji Medica College, Huazhong University of Science and Technology, No. 26, Shengli Street, Wuhan City, Hubei Province 430014, People's Republic of China. ${ }^{2}$ Center of Oncology, Renmin Hospital of Wuhan University, No.99, Zhangzhidong Road, Wuhan City, Hubei Province 430060, People's Republic of China.

Received: 12 May 2017 Accepted: 16 August 2017

Published online: 25 August 2017

\section{References}

1. Cazzola M. Introduction to a review series: the 2016 revision of the WHO classification of tumors of hematopoietic and lymphoid tissues. Blood. 2016; 127:2361-4.

2. Fonkem E, Lok E, Robison D, Gautam S, Wong ET. The natural history of intravascular lymphomatosis. Cancer Med. 2014;3:1010-24.

3. Pfleger $L$, Tappeiner J. On the recognition of systematized endotheliomatosis of the cutaneous blood vessels (reticuloendotheliosis?). Hautarzt. 1959;10:359-63.
4. Brunet V, Marouan S, Routy JP, Hashem MA, Bernier V, Simard R, et al. Retrospective study of intravascular large B-cell lymphoma cases diagnosed in Quebec: a retrospective study of 29 case reports. Medicine (Baltimore). 2017:96:e5985.

5. Hall JM, Meyers N, Andrews J. Hemophagocytosis-related (Asian variant) intravascular large B-cell lymphoma in a Hispanic patient: a case report highlighting a micronodular pattern in the spleen. Am J Clin Pathol. 2016;145:727-35.

6. Tajima S, Waki M, Yamazaki H, Nagata Y, Fukano H, Hossen MA, et al. Intravascular large B-cell lymphoma manifesting as cholecystitis: report of an Asian variant showing gain of chromosome 18 with concurrent deletion of chromosome 6q. Int J Clin Exp Pathol. 2014;7:8181-9.

7. Tahsili-Fahadan P, Rashidi A, Cimino PJ, Bucelli RC, Keyrouz SG. Neurologic manifestations of intravascular large B-cell lymphoma. Neurol Clin Pract. 2016;6:55-60.

8. Fonkem E, Dayawansa S, Stroberg E, Lok E, Bricker PC, Kirmani B, et al. Neurological presentations of intravascular lymphoma (IVL): meta-analysis of 654 patients. BMC Neurol. 2016;16:9.

9. Wilcox RA. Cutaneous B-cell lymphomas: 2016 update on diagnosis, riskstratification, and management. Am J Hematol. 2016;91:1052-5.

10. Diaz-Crespo F, Mollejo M. Intravascular large B-cell lymphoma in a kidney biopsy. Blood. 2016;127:2939.

11. Shigematsu Y, Matsuura M, Nishimura N, Tsuyama N, Takeuchi K, Terui Y, et al. Intravascular large B-cell lymphoma of the bilateral ovaries and uterus in an asymptomatic patient with at $(11 ; 22)(q 23 ; q 11)$ constitutional translocation. Intern Med. 2016;55:3169-74.

12. Li W, Lin W, Ma C, Zhang L, Sun H. A case of intravascular large B-cell lymphoma in the left adrenal and another tumor in the right adrenal detected by (18)F-FDG PET/CT. Hell J Nucl Med. 2016;19:57-9.

13. Stonecypher M, Yan Z, Wasik MA, LiVolsi V. Intravascular large B cell lymphoma presenting as a thyroid mass. Endocr Pathol. 2014;25:359-60.

14. Murase T, Yamaguchi M, Suzuki R, Okamoto M, Sato Y, Tamaru J, et al. Intravascular large B-cell lymphoma (IVLBCL): a clinicopathologic study of 96 cases with special reference to the immunophenotypic heterogeneity of CD5. Blood. 2007;109:478-85.

15. Hurlbeck S, Weidenthaler-Barth B, Butsch F. Early diagnosis of intravascular large B-cell lymphoma. J Dtsch Dermatol Ges. 2016;14:1146-8.

16. Wu F, Wang Z, Xing X, Yu M, Shi B. The value of 18F-FDG PET/CT in diagnostic procedure of intravascular large B-cell lymphoma presenting fever of unknown origin and pulmonary hypertension as an initial manifestation. Clin Nucl Med. 2016;41:506-7.

17. Higashiyama A, Komori T, Inada Y, Nakajima H, Narumi Y. Central Nervous System Involvement of Intravascular Large B-Cell Lymphoma on 18F-FDG PET/CT. Clin Nucl Med. 2017;"42(5):e258-e260.

18. lacobelli J, Spagnolo DV, Tesfai Y, Giardina TM, Chan JJ, Joske D, et al. Cutaneous intravascular anaplastic large T-cell lymphoma: a case report and review of the literature. Am J Dermatopathol. 2012;34:e133-8.

19. Alhumidi A. Cutaneous intravascular NK/T-cell lymphoma mimic panniculitis clinically, case report and literature brief review. Diagn Pathol. 2015;10:107.

20. Shimizu I, Ichikawa N, Yotsumoto M, Sumi M, Ueno M, Kobayashi H. Asian variant of intravascular lymphoma: aspects of diagnosis and the role of rituximab. Intern Med. 2007:46:1381-6.

21. Shimada K, Kinoshita T, Naoe T, Nakamura S. Presentation and management of intravascular large B-cell lymphoma. Lancet Oncol. 2009;10:895-902.

22. Adler NR, Sia CS, Polchleb C, Jane S, Aung AK. Intravascular large B cell lymphoma with haemophagocytic syndrome: a double lethal masquerade. Intern Med J. 2015;45:1310-2.

23. Singh D, Kapuria D, Nanua S, Gaur R. A case of de novo CD5+ disseminated intravascular large B-cell lymphoma presenting as multiorgan failure. Case Rep Hematol. 2016;2016:6239416.

24. Ubeda Romero A, Santonja C, Blanco Garcia A, Requena L, Rodriguez Pinilla SM. De novo CD5-positive diffuse large B-cell lymphoma: report of a case presenting with Cutaneous involvement and featuring extensive intravascular dissemination on postmortem examination. Int I Surg Pathol. 2016;24:763-8.

25. Tsuda M, Nakashima Y, Ikeda M, Shimada S, Nomura M, Matsushima T, et al. Intravascular large B-cell lymphoma complicated by anti-Neutrophil Cytoplasmic antibody-associated Vasculitis that was successfully treated with Rituximabcontaining chemotherapy. J Clin Exp Hematop. 2015;55:39-43.

26. Mitsutake A, Kanemoto T, Suzuki Y, Sakai N, Kuriki K. A case of intravascular large Bcell lymphoma that presented with recurrent multiple cerebral infarctions and followed an indolent course. Rinsho Shinkeigaku. 2015;55:101-6. 